\title{
EdITH FRÜNDT
}

Bibliographie zur Kunstgeschichte von Mecklenburg und Vorpommern 
DEUTSCHE AKADEMIE DER WISSENSCHAFTEN ZU BERLIN Arbeitsstelle für Kunstgeschichte

SCHRIFTEN ZUR KUNSTGESCHICHTE herausgegeben von

Richard Hamann $\dagger$ und Edgar Lehmann

Heft 8

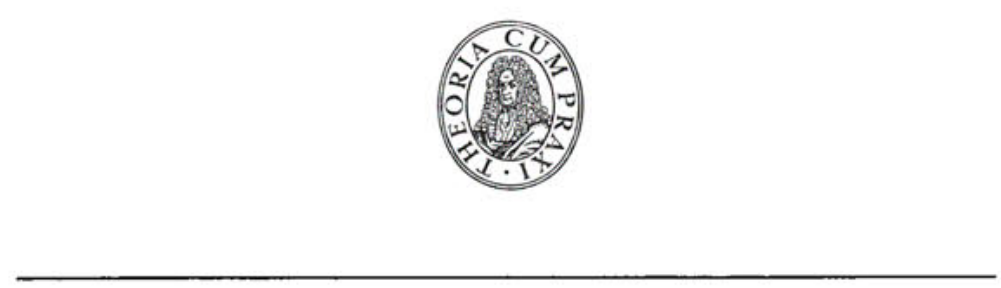

AKADEMIE-VERLAG · BERLIN

1962 
SCHRIFTEN ZUR KUNSTGESCHICHTE

\title{
Bibliographie zur Kunstgeschichte von
}

\section{Mecklenburg und Vorpommern}

\author{
VON \\ EDITH FRUNNDT
}

AKADEMIE-VERLAG - BERLIN

1962 
Erschienen im Akademie-Verlag GmbH, Berlin W 8, Leipziger Straße 3-4 Copyright 1962 by Akademie-Verlag $\mathrm{GmbH}$, Berlin

Lizenz-Nr. $202 \cdot 100 / 118 / 62$

Gesamtherstellung: Druckhaus „Maxim Gorki“", Altenburg

Bestellnummer: 2076/8 · ES I2 $\mathrm{C}_{2}$ 